\title{
Polyethylene glycol functionalized gold nanoparticles: the influence of capping density on stability in various media
}

\author{
Joanne Manson • Dhiraj Kumar • Brian J. Meenan • \\ Dorian Dixon
}

Published online: 27 April 2011

(C) The Author(s) 2011. This article is published with open access at Springerlink.com

\begin{abstract}
Thiol-terminated polyethylene glycol (PEG) is commonly used to functionalize the surface of gold nanoparticles (AuNPs) in order to improve their in vivo stability and to avoid uptake by the reticular endothelial system. Although it has been reported that AuNPs functionalized with tethered PEG are stable in biological media, the influence of chain density remains unclear. This study investigates the influence of PEG capping density on the stability of washed and dried AuNPs in: water, phosphate-buffered saline solution (PBS), phosphate-buffered saline solution containing bovine serum albumin (PBS/BSA), and dichloromethane (DCM). PEG coating had a dramatic effect on stability enabling stable suspensions to be produced in all the media studied. A linear relationship was observed between capping density and stability in water and DCM with a somewhat lower stability observed in PBS and PBS/BSA. A maximum PEG loading level of $\sim 14 \mathrm{wt} . \%$ was achieved, equivalent to a PEG surface density of $\sim 1.13$ chains $/ \mathrm{nm}^{2}$.
\end{abstract}

Keywords Gold nanoparticle · Drying · Redispersion in media $\cdot$ Thiolated poly(ethylene glycol)

\section{Introduction}

Metallic nanoparticles are the subject of a concerted international research effort as novel platforms for the

Electronic supplementary material The online version of this article (doi:10.1007/s13404-011-0015-8) contains supplementary material, which is available to authorized users.

J. Manson · D. Kumar • B. J. Meenan · D. Dixon $(\triangle)$

Nanotechnology and Integrated Bioengineering Centre,

University of Ulster,

Jordanstown, Shore Road,

Newtownabbey BT37 0QB Northern Ireland, UK

e-mail: d.dixon@.ulster.ac.uk target-specific delivery of therapeutic agents. Gold nanoparticles (AuNPs) in particular are an excellent candidate for drug delivery vehicles due to their unique physical and chemical properties, enabling the transport and subsequent release of therapeutic payloads such as drugs or genetic materials to specific tissue sites. Their advantages include ease of synthesis in a range of monodisperse sizes from 1 to $150 \mathrm{~nm}$, an essentially inert and non-toxic nature and an ability to be readily functionalized with targeting ligands and drugs. Payloads can be subsequently released at the required site by way of their photo-physical properties or by intercellular glutathione levels [1-3]. Active and passive targeting to a disease site such as a tumor can be achieved by attaching ligands such as tumor necrosis factor- $\alpha$, or proteins onto the nanoparticles, or through size and charge effects $[3,4]$. A wide range of molecules can be tethered onto the AuNP surface by means of a thiol (SH) group. AuNPs are also readily imaged which enables direct tracking of their fate within cells.

Gold nanoparticles can be synthesized in a range of shapes and sizes such as rods, triangles, cubes and wires [5-9]. They are generally synthesized chemically using sodium borohydride, sodium citrate or, more recently, hydroquinone reduction of a chloroauric acid $\left(\mathrm{HAuCl}_{4}\right)$ solution [10-16]. The ratio of gold salt to reducing agent, type of reducing agent, and temperature play a critical role in determining the size and shape of the nanoparticles [17, 18]. In the case of sodium citrate reduction, the citrate acts as a loosely bound capping agent stabilizing the particles. Tetradecane, octadecane or dodecane thiols are often used as capping agents to stabilize AuNPs produced via sodium borohydride reduction $[19,20]$. The biological response can be altered using capping agents such as polyethylene glycol (PEG) also referred to as polyethylene oxide (PEO) [20, 21], mercaptosuccinic acid, various proteins [21] or other biomolecules [2]. Such functionalization has led to an 
extensive research effort investigating AuNPs as carriers for a range of biomolecules and drugs [22-27].

While it is known that functionalizing AuNPs with PEG increases stability, both in vivo and in vitro, the influence of capping density and the effect that washing and drying has on resuspension in a range of media including organic solvents has not been previously reported. This study reveals the effect of PEG surface density on the aggregation behaviour of AuNPs in: water, an organic solvent dichloromethane (DCM) and the model biological media, phosphate-buffered saline (PBS) and PBS with bovine serum albumin (BSA).

\section{Materials and methods}

\section{Materials}

Prior to synthesis, all the glassware was washed with aqua regia, rinsed with distilled water and then dried overnight at $80-100^{\circ} \mathrm{C}$. Magnetic flees were first sonicated in ethanol and then in de-ionized (DI) water for $15 \mathrm{~min}$ and dried before use. Chloroauric acid $\left(\mathrm{HAuCl}_{4} \cdot 3 \mathrm{H}_{2} \mathrm{O}\right)$, PEG $\left(5,000 \mathrm{M}_{\mathrm{w}}\right)$ and sodium citrate were obtained from Sigma Aldrich UK. The 0.01 wt. $\%$ chlorauric acid and 1 wt. $\%$ sodium citrate solutions were prepared using DI water, PBS, DCM (99.9\%) and PBS with $0.05 \mathrm{wt} \%$ BSA, which were also obtained from Sigma Aldrich and used as received.

Synthesis and washing of gold nanoparticles

Chloroauric acid solution ( $200 \mathrm{ml}$ of $0.01 \mathrm{wt} . \%$ ) was heated to a rolling boil and refluxed in a 500-ml-round-bottom flask using a temperature-controlled hot plate with continuous stirring [4]. A 4.5-ml aliquot of 1 wt.\% sodium citrate solution was then added to the boiling chloroauric acid solution, and the heating was continued under reflux for $15 \mathrm{~min}$ to enable complete reaction. The solution was then allowed to cool to room temperature with continuous stirring yielding citrate-capped AuNPs. In order to produce PEGcapped AuNPs, various concentrations (3.6, 8.4, 16.8 and $25.2 \mu \mathrm{g}$ per $\mathrm{ml}$ of as synthesized AuNP suspension) of 5,000 $\mathrm{M}_{\mathrm{w}}$ PEG were added to the 'as synthesized' AuNP solutions at room temperature. After the required amount of PEG was added, the solution was stirred at room temperature for $2 \mathrm{~h}$ to allow for complete exchange of the citrate molecules with PEG. The AuNP solutions were then centrifuged using a Contifuge 17RS, Heraeus SEPATECH at $10,000 \mathrm{rpm}$ for $90 \mathrm{~min}$ in $10 \mathrm{ml}$ batches [28]. Of the supernatant, $9.9 \mathrm{ml}$ was then decanted, leaving the AuNP pellet at the bottom of the centrifuge tube. The volume was then made back up to $10 \mathrm{ml}$ by adding $9.9 \mathrm{ml}$ of DI water and agitated. This centrifugal washing process was repeated again to remove any unattached PEG or other reactants.
Dispersion of AuNPs in relevant media

After washing, both the citrate- and PEG-capped AuNPs were dried at $60^{\circ} \mathrm{C}$ for 3 days. The dried samples were sonicated for $15 \mathrm{~min}$ in a sonic bath and then redispersed in the required media at the original 'as synthesized' concentrations and further sonicated for $30 \mathrm{~min}$. The media investigated were deionised $\mathrm{H}_{2} \mathrm{O}$, PBS $(137 \mathrm{mM} \mathrm{NaCl}, 2.7 \mathrm{mM} \mathrm{KCl}, 10 \mathrm{mM}$ PBS at pH 7.4), PBS containing 0.05 wt. $\%$ BSA and DCM (99.9\%). Dispersions of all five AuNP types investigated (one capped with citrate and four densities of PEG) were then prepared in each of the four media of interest.

Physical measurements

UV-Visible spectra were recorded using a Perkin Elmer Lambda 11 in the range $400-600 \mathrm{~nm}$ at $0.1 \mathrm{~nm}$ increments, using $1 \mathrm{ml}$ of sample solution in a 1.4-ml glass spectrophotometer cuvette (Sigma Aldrich). The UV-vis spectra of the 'as synthesized' sample of each type before washing, drying and redisperion were used as the reference spectra for the subsequent calculation of relative absorbance. After redispersion, UV-vis spectra were taken at five time points over a 24-h period, with three replicate spectra recorded.

Citrate and PEG $(16.8 \mu \mathrm{g} / \mathrm{ml})$-capped AuNP samples were dried onto carbon-coated copper grids and imaged using a JEOL 2100 Transmission Electron Microscope at $200 \mathrm{kV}$. For SEM, gold nanoparticle solutions were dried onto titanium metal stubs and imaged at $20 \mathrm{kV}$ using a FEI Helios NanoLab.

DLS was conducted using Nano ZetaSizer ZS Series. For size determination, $3 \mathrm{ml}$ of each sample was measured in a disposable cuvette. For Zeta potential, samples were analysed using a standard zeta cuvette. The samples were analysed three times at $25^{\circ} \mathrm{C}$.

Infrared spectra were obtained using a BIORAD FTS 3000MX Excalibur series spectrometer fitted with a DRIFTS accessory. Fifty millilitres of each sample type was centrifuged, and the pellets dried in a Teflon-coated dish at room temperature. The dried samples were then mixed with $\mathrm{KBr}$ to produce a fine powder which was pressed into discs. All spectra were recorded at a resolution of $4 \mathrm{~cm}^{-1}$ over a wave number range of $400-4,000 \mathrm{~cm}^{-1}$.

TGA was carried out using a TGA Q600 (TA Instruments) between $24^{\circ} \mathrm{C}$ and $800^{\circ} \mathrm{C}$ at $10^{\circ} \mathrm{C} / \mathrm{min}$ under a nitrogen atmosphere flowing at $40 \mathrm{ml} / \mathrm{min}$. Each $\sim 5-\mathrm{mg}$ sample was prepared by drying the washed AuNP solutions. The amount of PEG attachment was calculated as the percentage weight loss occurring between $302^{\circ} \mathrm{C}$ and $450^{\circ} \mathrm{C}$, which corresponds to the degradation of the PEG. The $1.8 \%$ weight loss recorded for the citrate-capped AuNPs over this temperature range was taken into account when calculating the levels of PEG attachment. 


\section{Results and discussion}

\section{Characterization of the AuNPs}

UV-vis absorbance spectra for citrate-capped AuNPs displayed a characteristic peak at $519.4 \mathrm{~nm}$ compared to 519.5-520.6 nm for PEG-capped AuNPs. This peak relates to the surface plasmon, and an increase in intensity was observed as PEG functionalization density increased. This effect has been reported previously [29].

From Fig. 1, it can be seen that the AuNPs are approximately spherical and reasonably monodisperse (additional TEM and SEM images are available in online resource 1 and 2). However, some of the particles display a somewhat faceted shape as a result of their nanocrystalline nature. From the TEM images, an average particle diameter and standard deviation of 17.8 and $1.7 \mathrm{~nm}$, respectively, were recorded for the citrate-capped AuNPs, compared to 17.9 and $1.9 \mathrm{~nm}$, respectively, for the AuNPs functionalized with $16.8 \mu \mathrm{g} / \mathrm{ml}$ of PEG. It was expected that no significant difference in size between citrate- and PEG-capped AuNP would be observed via TEM, as only the gold core is visible at the acceleration voltage used [24].

DLS measurements of the AuNPs reported as volume average diameters are shown in Table 1 (additional data are available in online resource 4, 5 and 6). TEM and DLS measurements reported similar average diameters for the citrate-capped AuNPs at 17.8 and $17.3 \mathrm{~nm}$, respectively. However, the average diameter measured by DLS increases as a result of PEG attachment because the technique measures equivalent hydrodynamic radius and is sensitive to the effect of PEG capping. Hall et al. reported a change in AuNP average diameter measured using DLS from 28.2 to $48.5 \mathrm{~nm}$ with the addition of a PEG coating. Takae et al. observed an increase in nanoparticle diameter from 20 to $33.3 \mathrm{~nm}$ after functionalization with $6,000 \mathrm{M}_{\mathrm{w}}$ PEG, whereas Arinda et al. found that functionalization with 5,000 $\mathrm{M}_{\mathrm{w}}$ PEG increased AuNP diameter from 50 to $89 \mathrm{~nm}$ [30]. In the case of AuNP functionalized with $550 \mathrm{M}_{\mathrm{w}}$ PEG, Oh et al. reported that the average diameter from DLS was some 6-9 $\mathrm{nm}$ higher than that measured using TEM. The end to end length of $5,000 \mathrm{M}_{\mathrm{w}}$ based on a Gaussian random coil model is $\sim 8 \mathrm{~nm}$ compared to $\sim 40 \mathrm{~nm}$ for a zigzag model [31]. Referring to Table 1, the observed increase in diameter from 17.3 to $36.4 \mathrm{~nm}$ as a result of PEG attachment equates to a capping thickness of $\sim 9.5 \mathrm{~nm}$. Based on this data, we suggest that the PEG chains have adopted a somewhat extended random coil conformation.

Typical FTIR spectra of washed and dried citrate- and PEG-capped AuNPs are shown in Fig. 2. The additional
Fig. 1 TEM images and accompanying histograms for a citrate capped and b $16.8 \mu \mathrm{g} / \mathrm{ml}$ PEG
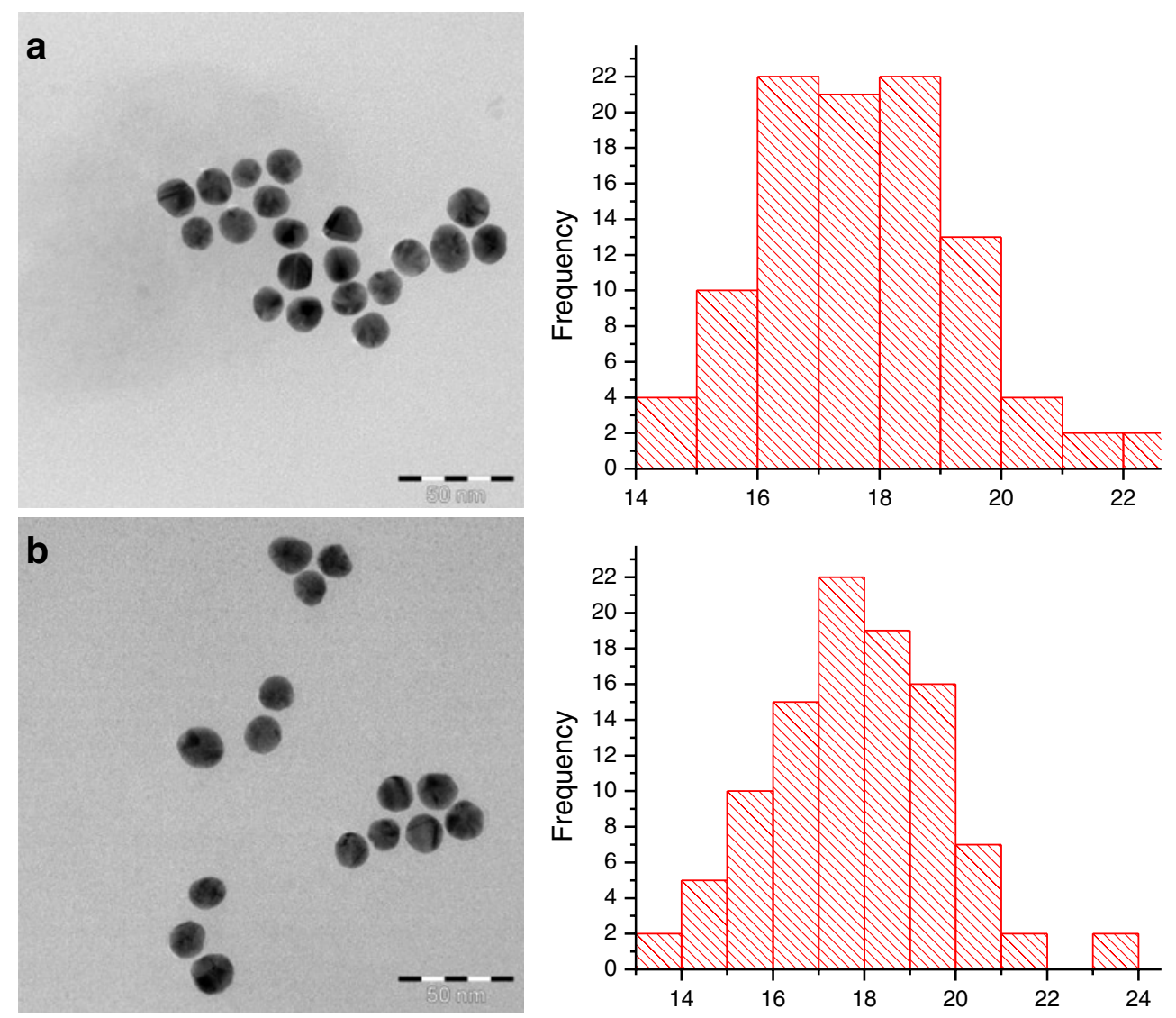
Table 1 Effect of thiolated PEG on the characteristics of AuNPs

\begin{tabular}{lllll}
\hline $\begin{array}{l}\text { Amount of } \\
\text { PEG }(\mu \mathrm{g} / \mathrm{ml})\end{array}$ & $\begin{array}{l}\text { PEG weight\% } \\
\text { from TGA }\end{array}$ & $\begin{array}{l}\text { Diameter (nm) } \\
\text { from TEM }\end{array}$ & $\begin{array}{l}\text { DLS volume average } \\
\text { diameter }(\mathrm{nm})\end{array}$ & $\begin{array}{l}\text { Polydispersity } \\
\text { index (PDI) }\end{array}$ \\
\hline 0 (citrate capped) & 0.0 & 17.8 & 17.3 & 0.282 \\
3.4 & 2.6 & & 29.3 & 0.387 \\
8.4 & 6.8 & 17.9 & 32.6 & 0.249 \\
16.8 & 12.7 & & 32.8 & 0.249 \\
25.2 & 13.8 & & 36.4 & 0.297 \\
\hline
\end{tabular}

peaks present in the PEG-capped spectrum are assigned as follows: $2,850-3,000 \mathrm{~cm}^{-1}$ (- $\mathrm{CH}_{2}$ stretching), $1,631 \mathrm{~cm}^{-1}$ (N-H bending), $1,660 \mathrm{~cm}^{-1}$ ( $\mathrm{C}=\mathrm{O}$ stretching), $1,380 \mathrm{~cm}^{-1}$ (C-H bending; $-\mathrm{CH}_{2}$ and $\left.-\mathrm{CH}_{3}\right), 1,100 \mathrm{~cm}^{-1}$ (C-O-C stretching) and $600-900 \mathrm{~cm}^{-1}$ (N-H wagging) and confirm the presence of bound PEG [32].

Figure 3 shows the TGA traces for AuNPs capped with citrate, the varying amounts of PEG and 'as received' $5,000 \mathrm{M}_{\mathrm{w}}$ thiolated PEG. In the case of 'as received' PEG, weight loss occurs between $300^{\circ} \mathrm{C}$ and $450^{\circ} \mathrm{C}$ relating to the thermal degradation of the polymer. This weight loss step is also observed in the TGA of the PEGcapped AuNPs, but not the citrate-capped particles, with greater mass loss indicative of higher levels of PEG attachment. The weight percentages of bound PEG resulting from the different concentrations of PEG used during synthesis calculated using the TGA results are shown in Table 1.

The relationship between the concentration of PEG used during synthesis, the amount bound to AuNPs surface after washing and that remaining in the supernatant is shown in Fig. 4. At low concentrations, all the PEG added adheres to the AuNPs, with increasing amounts remaining in the supernatant at higher addition rates as the AuNP surface

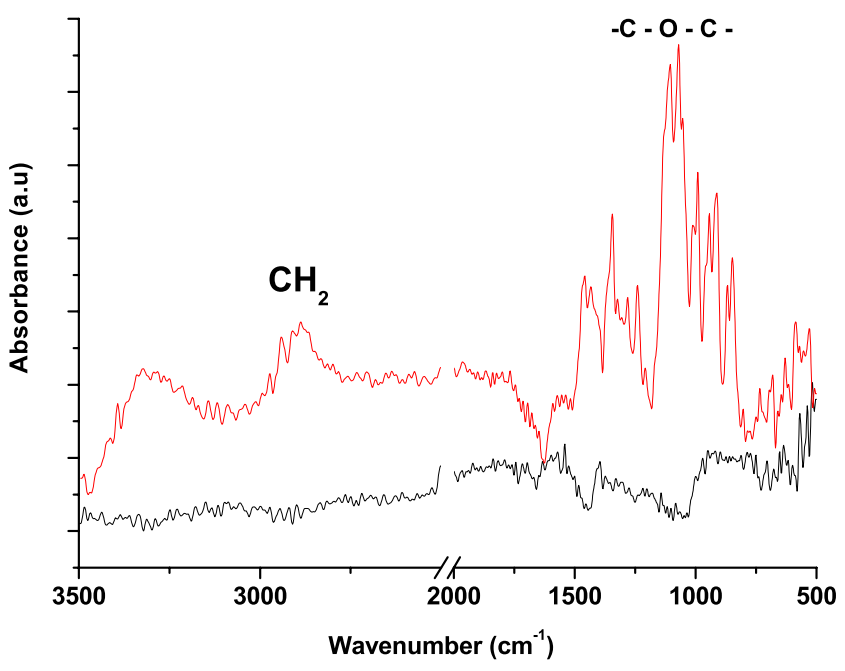

Fig. 2 FTIR spectra for citrate (bottom) and PEG $(16.8 \mu \mathrm{g} / \mathrm{ml}$; top $)$ capped AuNPs becomes saturated. This saturation is apparent at a PEG addition rate of $16.8 \mu \mathrm{g} / \mathrm{ml}$ with a maximum PEG loading achievable of $\sim 14 \mathrm{wt} . \%$. This corresponds to approximately 1,140 thiol-terminated PEG molecules per AuNP giving a chain density of 1.13 chains $/ \mathrm{nm}^{2}$ (assuming a spherical particle $17.9 \mathrm{~nm}$ in diameter). This is significantly below the theoretical density for a close-packed helical layer of PEG of which is $\sim 5$ chains $/ \mathrm{nm}^{2}$ [33]. In previously published work on PEG attachment to planer gold surfaces, Unsworth et al. found a maximum density of 0.34 chains $/ \mathrm{nm}^{2}$ for 5,000 $\mathrm{M}_{\mathrm{w}}$ PEG while Anne et al. reported a maximum of 0.1 chains $/ \mathrm{nm}^{2}$ for $3,400 \mathrm{M}_{\mathrm{w}}[33,34]$. Denser packing is achievable on the curved surface of AuNPs compared to that on a flat substrate due to a reduction in steric hindrance as the free end of the PEG molecules are less confined.

DLS measurement found that the surface charge ( $\zeta$ potential) for the citrate-capped AuNP was $-39.5 \mathrm{mV}$ compared to $-13.9 \mathrm{mV}$ for the highest PEG loading, a result of steric shielding.

\section{Colloid stability tests}

The relative UV-vis absorbance values of AuNPs resuspended in $\mathrm{H}_{2} \mathrm{O}$, PBS, PBS-BSA and DCM over a 24$\mathrm{h}$ period are shown in Fig. 5. While citrate-capped AuNP samples are stable for extended periods in the 'as synthesized' state, it is clear from Fig. 5 that it is not possible to wash (by centrifugation), dry and successfully resuspend these AuNPs. However, stable dispersions of the citrate-capped AuNPs can be produced in PBS and PBSBSA (results not shown) by making up the media using the 'as synthesized' AuNPs. This has also been previously observed in literature [35-39]. The negligible absorbance values recorded for washed and dried citrate-capped AuNP after resuspension in the four media indicate that aggregation has occurred. Increasing levels of PEG attachment leads to a dramatic enhancement in stability, with stable suspensions of washed and dried AuNPs achieved in all media. In the case of $\mathrm{H}_{2} \mathrm{O}$ and DCM, an approximately linear relationship is observed between relative UV-vis absorbance after redispersion and wt.\% of added PEG (a graph of relative absorbance versus PEG attachment is 
Fig. 3 TGA of AuNP with various concentrations of PEG and 'as-received' PEG

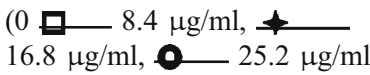
$16.8 \mu \mathrm{g} / \mathrm{ml}, \boldsymbol{O} 25.2 \mu \mathrm{g} / \mathrm{ml}$ PEG-SH)

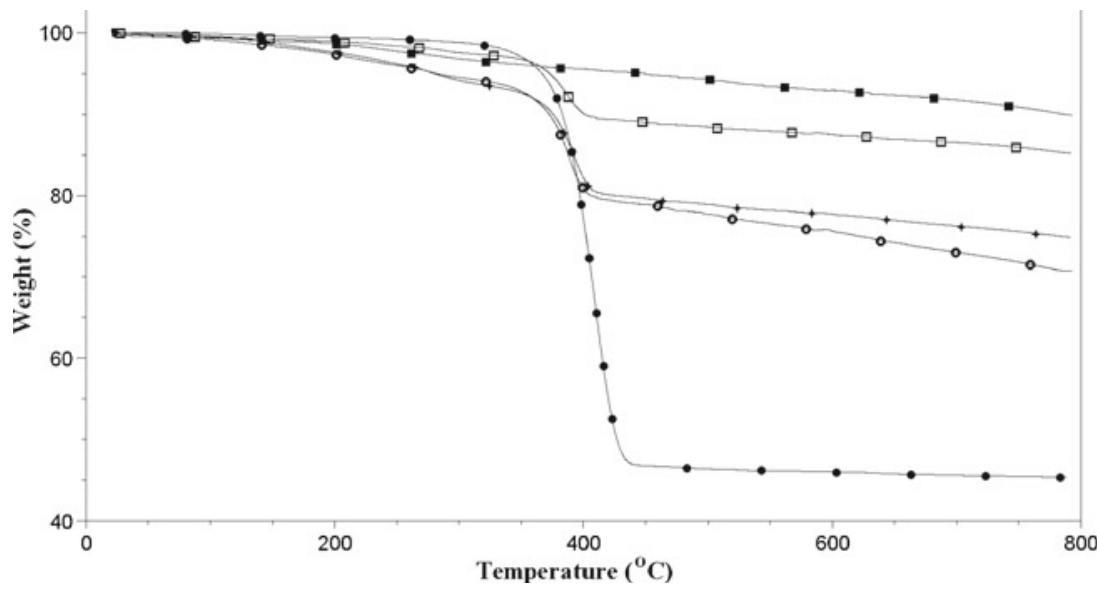

available in online resource 7). At a PEG addition rate of $25.2 \mu \mathrm{g} / \mathrm{ml}$, the relative absorbance of 0.88 and 0.9 is observed for DCM and $\mathrm{H}_{2} \mathrm{O}$ after washing, drying and redispersion. This indicates that the majority of the AuNPs exist as single stable particles after washing, drying and resuspension. While Takae et al. have observed that PEGcoated AuNPs can be successfully resuspended after centrifugation, the data shown in Fig. 5 illustrate that resuspension of functionalized AuNPs is possible after multiple centrifuging cycles and drying. Drying is necessary before resuspending the particles in non-aqueous media. Zheng et al. reported the ability to dry and redisperse $3 \mathrm{~nm}$ tetra(ethylene glycol) monolayer protected gold nanoparticles in organic solvents such as acetone, methanol, DMSO, $\mathrm{CHCl}_{3}, \mathrm{DMF}$ and THF by direct synthesis in a mixture of water and methanol [40]. While Otsuka et al. reported that $8.9 \mathrm{~nm}$ PEG-capped gold nanoparticles are also stable in PBS, BSA and PBS containing 2\% serum after centrifugation [41].
It should be noted that the increase in absorbance values observed in Fig. $5 d$ is due to solvent evaporation over the 24h study. It was observed that sonicating the dried AuNPs in a sonic bath after drying for $15 \mathrm{~min}$ followed by a further sonication for $30 \mathrm{~min}$ after adding the media led to enhanced dispersion. From Fig. 5c, it can also be seen that the introduction of BSA improves the stability of the AuNPs functionalized with $3.4 \mu \mathrm{g} / \mathrm{ml}$ of PEG. It has been previously reported that BSA binds to AuNPs [30, 42]. The observation here that BSA does not affect the stability of AuNPs with denser PEG coatings may be a result of the influence of any BSA attachment being masked by the effect of the higher levels of PEG and the denser coating impeding protein attachment. AuNP stability in PBS and PBS-BSA was somewhat lower than that observed in $\mathrm{H}_{2} \mathrm{O}$ and DCM due to the aggregating influence of $\mathrm{NaCl}$. A critical salt concentration study found that AuNPs functionalized with 16.8 and $25.2 \mu \mathrm{g} / \mathrm{ml}$ of PEG remain stable in $1 \mathrm{M} \mathrm{NaCl}$,
Fig. 4 PEG concentration remaining in supernatant after first centrifugation and bound to the AuNP surface measured by UV-vis and TGA, respectively. - - $-P E G$ in supernatant via UV-vis, - - -weight (\%)

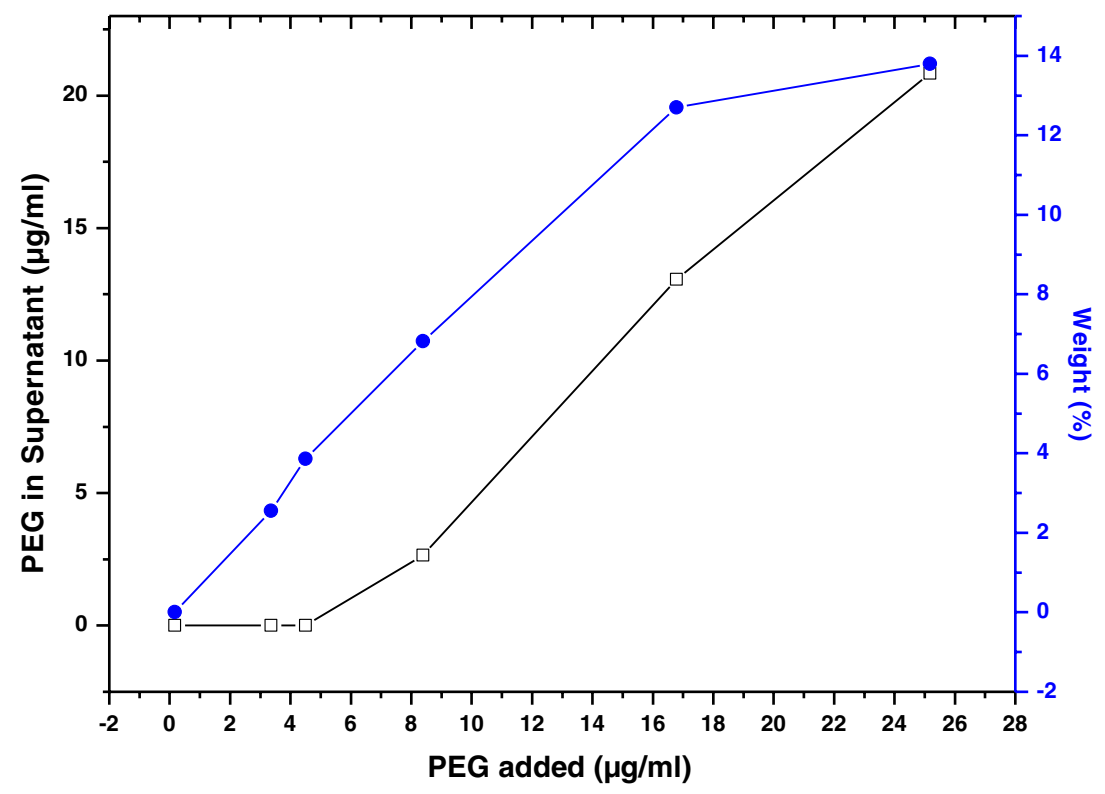


a

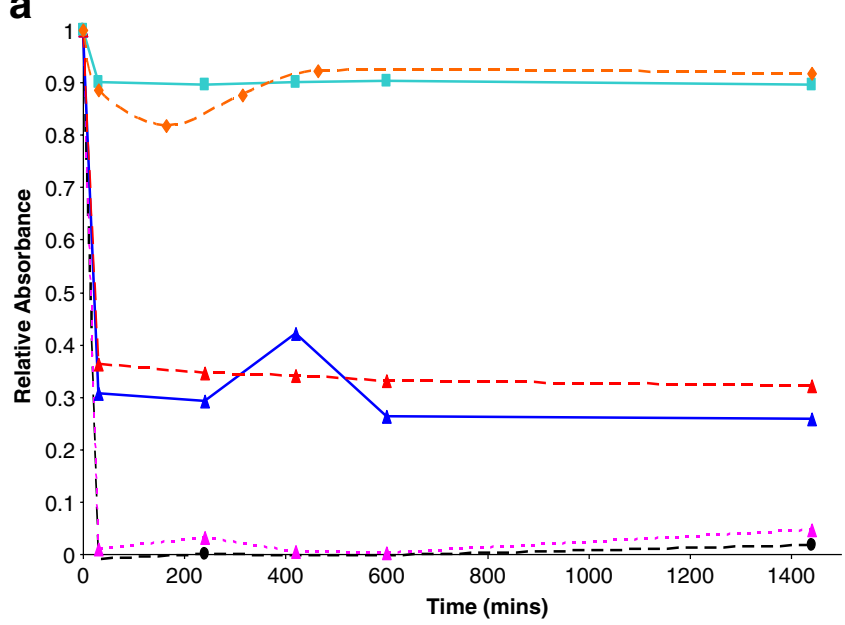

C

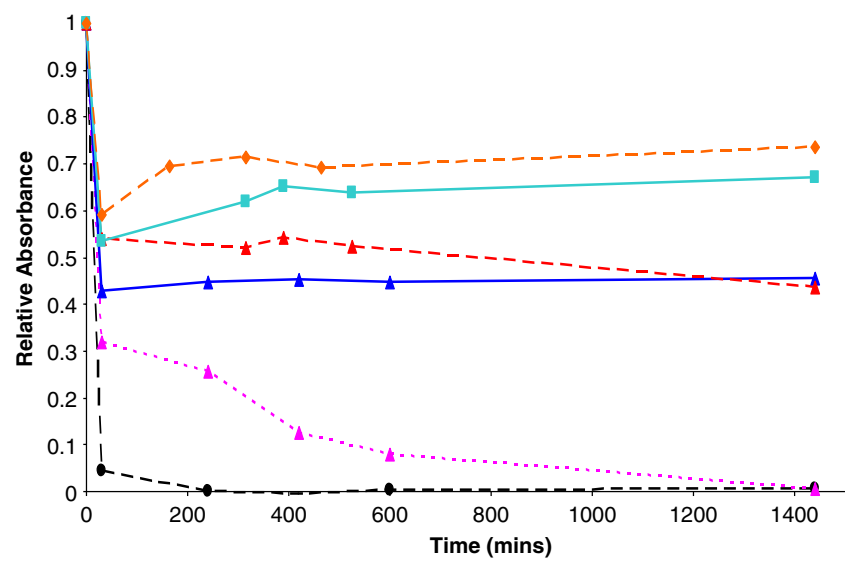

b

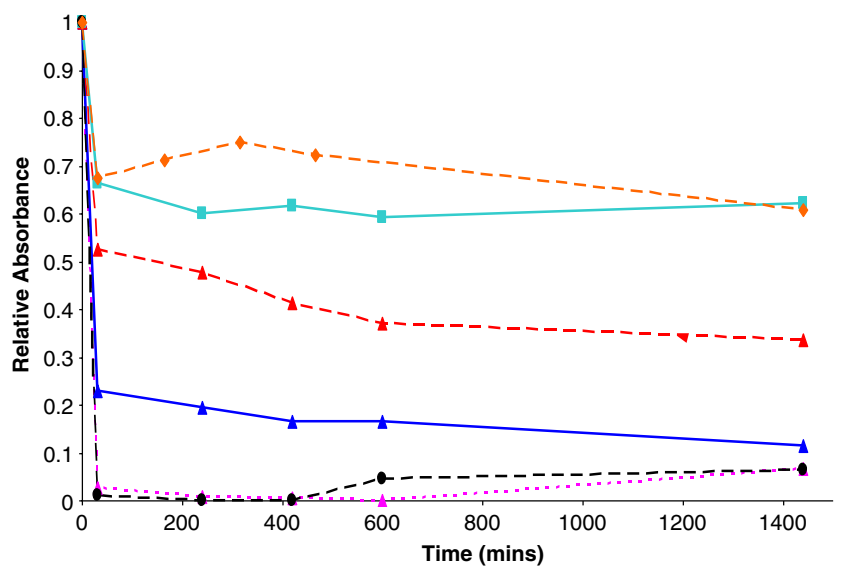

d

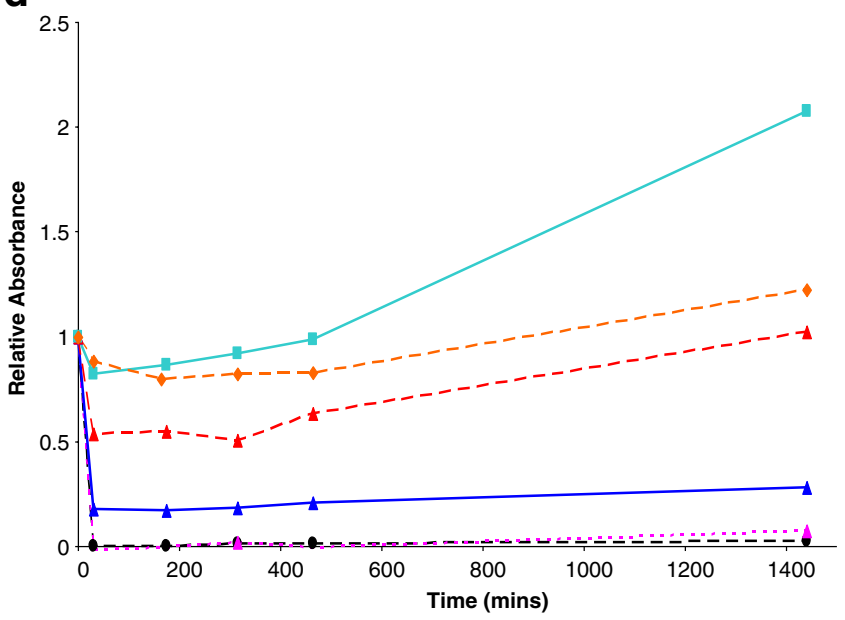

Fig. 5 Relative absorbance of AuNPs with different amounts of PEG redispersed in various media over 24 h. a $\mathrm{H}_{2} \mathrm{O}$, b PBS, c PBS-BSA, d

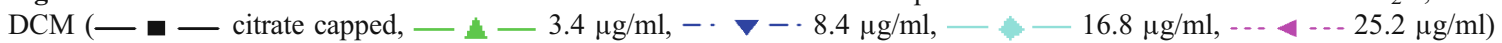

which is a value well in excess of the normal physiological salt concentration $(0.15 \mathrm{M})$.

\section{Conclusions}

Stable dispersions of washed and dried PEG coated gold nanoparticles were achieved in water, PBS, PBS containing BSA and DCM. PEG-coated AuNPs were also found to be stable at salt concentrations of $0.15-1 \mathrm{M}$, whereas citratecapped AuNPs aggregated immediately under such conditions. In the case of redispersion in DCM and $\mathrm{H}_{2} \mathrm{O}$, there is an approximately linear relationship between the amount of PEG attachment and stability. Lower levels of stability are observed in highly PEG-functionalized AuNPs after redispersion in PBS and PBS/BSA compared to DCM and $\mathrm{H}_{2} \mathrm{O}$ due to the effect of $\mathrm{NaCl}$. It was observed that saturation of the AuNP surface with tethered PEG occurs at a PEG loading level of $\sim 14 \mathrm{wt} \%$, equivalent to $\sim 1.13$ chains $/ \mathrm{nm}^{2}$, and that the PEG chains adopt an elongated random coil configuration. This is well below the maximum theoretical packing density of $\sim 5$ chains $/ \mathrm{nm}^{2}$. Significant levels of stability enhancement were achieved at lower loading levels allowing for the co-functionalization of AuNPs with drugs or targeting groups for applications in assays and drug carrier systems.

Acknowledgements The work was funded by the Department of Employment and Learning (DEL) as part of the Collaborative Centre for Functional Biomaterials (Project UU005). The authors wish to acknowledge Thomas Dooher who conducted the TEM analysis under the Science Foundation Ireland funded National Access Program (NAP Project 242). DLS measurements were conducted with the assistance of Yuri Rochev in NUI Galway.

Open Access This article is distributed under the terms of the Creative Commons Attribution License which permits any use, distribution and reproduction in any medium, provided the original author(s) and source are credited. 


\section{References}

1. Hong R et al (2006) Glutathione-mediated delivery and release using monolayer protected nanoparticle carriers. J Am Chem Soc 128(4):1078-1079

2. Ghosh P et al (2008) Gold nanoparticles in delivery applications. Adv Drug Deliv Rev 60(11):1307-1315

3. Sonavane G, Tomoda K, Makino K (2008) Biodistribution of colloidal gold nanoparticles after intravenous administration: effect of particle size. Colloids Surf B 66(2):274-280

4. Kim D et al (2007) Antibiofouling polymer-coated gold nanoparticles as a contrast agent for in vivo X-ray computed tomography imaging. J Am Chem Soc 129(24):7661-7665

5. Jin $\mathrm{R}$ et al (2003) Controlling anisotropic nanoparticle growth through plasmon excitation. Nature 425(6957):487-490

6. Ah CS et al (2005) Size-controlled synthesis of machinable single crystalline gold nanoplates. Chem Mater 17(22):5558-5561

7. Sau TK, Murphy CJ (2004) Room temperature, high-yield synthesis of multiple shapes of gold nanoparticles in aqueous solution. J Am Chem Soc 126(28):8648-8649

8. Sun Y, Xia Y (2002) Shape-controlled synthesis of gold and silver nanoparticles. Science 298(5601):2176-2179

9. Wijaya A et al (2008) Selective release of multiple DNA oligonucleotides from gold nanorods. ACS Nano 3(1):80-86

10. Wagner FE et al (2000) Before striking gold in gold-ruby glass. Nature 407(6805):691-692

11. Higby GJ (1982) Gold Bulletin 15:130

12. Grabar $\mathrm{KC}$ et al (1995) Preparation and characterization of $\mathrm{Au}$ colloid monolayers. Anal Chem 67(4):735-743

13. Paciotti GF, Kingston DG, Tamarkin L (2006) Colloidal gold nanoparticles: a novel nanoparticle platform for developing multifunctional tumor-targeted drug delivery vectors. Drug Dev Res 67(1):47-54

14. Turkevich J, Stevenson PC, Hillier J (1951) Nucleation and growth process in the synthesis of colloidal gold. Discuss Faraday Soc 11:55-75

15. Frens G (1973) Controlled nucleation for the regulation of the particle size in monodisperse gold suspensions. Nature Phys Sci 241:20-22

16. Perrault SD, Chan WCW (2009) Synthesis and surface modification of highly monodispersed, spherical gold nanoparticles of 50-200 nm. J Am Chem Soc 131(47):17042-17043

17. Brown KR, Walter DG, Natan MJ (1999) Seeding of colloidal Au nanoparticle solutions. 2. Improved control of particle size and shape. Chem Mater 12(2):306-313

18. Kimling $\mathrm{J}$ et al (2006) Turkevich method for gold nanoparticle synthesis revisited. J Phys Chem B 110(32):15700-15707

19. Martin $\mathrm{MN}$ et al (2010) Charged gold nanoparticles in non-polar solvents: 10-min synthesis and 2D self-assembly. Langmuir 26 (10):7410-7417

20. Juillerat-Jeanneret L (2008) The targeted delivery of cancer drugs across the blood-brain barrier: chemical modifications of drugs or drug-nanoparticles? Drug Discov Today 13(23-24):1099-1106

21. Chen S, Kimura K (1999) Synthesis and characterization of carboxylate-modified gold nanoparticle powders dispersible in water. Langmuir 15(4):1075-1082

22. Muthu MS, Singh S (2009) Targeted nanomedicines: effective treatment modalities for cancer, AIDS and brain disorders. Nanomedicine 4(1):105-118

23. Duguet E et al (2006) Magnetic nanoparticles and their applications in medicine. Nanomedicine 1(2):157-168
24. Hall JB et al (2007) Characterization of nanoparticles for therapeutics. Nanomedicine 2(6):789-803

25. Zhang D et al (2009) Gold nanoparticles can induce the formation of protein-based aggregates at physiological $\mathrm{pH}$. Nano Lett 9 (2):666-671

26. Brust $M$ et al (1994) Synthesis of thiol derivatised gold nanoparticles in a two phase liquid/liquid system. J Chem Soc Chem Commun 7:801

27. Rao CNR, Müller A, Cheetham AK (2005) Nanomaterials - an introduction. In: Rao CNR, Müller A, Cheetham AK (eds) The chemistry of nanomaterials. Wiley-VCH Verlag $\mathrm{GmbH} \& \mathrm{Co}$. KGaA, Weinheim, FRG, pp 1-11

28. Roca M et al (2009) Linear assembly of gold nanoparticle clusters via centrifugation. Langmuir 26(3):2035-2041

29. Oh KS et al (2008) Gold/chitosan/pluronic composite nanoparticles for drug delivery. J Appl Polym Sci 108(5):3239-3244

30. Arnida A et al (2011) Geometry and surface characteristics of gold nanoparticles influence their biodistribution and uptake by macrophages. Eur J Pharm Biopharm 77:417-423

31. Dunn AS (1994) Introduction to physical polymer science (2nd edition). By L. H. Sperling, New York, 1992 John Wiley \& Sons Inc. John Wiley \& Sons Inc., New York, 1992. pp. xxvii + 594, price $£ 55.00$. ISBN 0-471-53035-2. Polymer International 33(2):233

32. Asadishad B, Vossoughi M, Alamzadeh I (2010) In vitro release behavior and cytotoxicity of doxorubicin-loaded gold nanoparticles in cancerous cells. Biotechnology Letters 32(5):649-654

33. Anne AS, Demaille C, Moiroux J (2002) Terminal attachment of polyethylene glycol (PEG) chains to a gold electrode surface. Cyclic voltammetry applied to the quantitative characterization of the flexibility of the attached PEG chains and of their penetration by mobile PEG chains. Macromolecules 35(14):5578-5586

34. Unsworth LD, Sheardown H, Brash JL (2005) Polyethylene oxide surfaces of variable chain density by chemisorption of PEO-thiol on gold: adsorption of proteins from plasma studied by radiolabelling and immunoblotting. Biomaterials 26(30):5927-5933

35. Paciotti GF et al (2004) Colloidal gold: a novel nanoparticle vector for tumor directed drug delivery. Drug Deliv 11(3):169-183

36. Housni A et al (2008) Monodisperse protein stabilized gold nanoparticles via a simple photochemical process. J Phys Chem C 112(32):12282-12290

37. Zhang $G$ et al (2009) Influence of anchoring ligands and particle size on the colloidal stability and in vivo biodistribution of polyethylene glycol-coated gold nanoparticles in tumor-xenografted mice. Biomaterials 30(10):1928-1936

38. Zhang Q et al (2010) Uptake of gold nanoparticles in murine macrophage cells without cytotoxicity or production of proinflammatory mediators. Nanotoxicology. doi:10.3109/17435390. 2010.512401, 0(0):1-12

39. Shujuan H et al (2002) Optical spectroscopic studies of the dispersibility of gold nanoparticle solutions. J Appl Phys 92 (12):7486-7490

40. Zheng M, Davidson F, Huang X (2003) Ethylene glycol monolayer protected nanoparticles for eliminating nonspecific binding with biological molecules. J Am Chem Soc 125 (26):7790-7791

41. Otsuka $\mathrm{H}$ et al (2001) Quantitative and reversible lectin-induced association of gold nanoparticles modified with $\alpha$-lactosyl- $\omega$ mercapto-poly(ethylene glycol). J Am Chem Soc 123(34):8226-8230

42. Takae $\mathrm{S}$ et al (2005) Ligand density effect on biorecognition by PEGylated gold nanoparticles: regulated interaction of RCA120 lectin with lactose installed to the distal end of tethered PEG strands on gold surface. Biomacromolecules 6(2):818-824 